\title{
Quantificação de fenóis totais presentes nas cascas de Trichillia catigua A. Juss (Meliaceae)
}

\author{
RABELO, D.S.'; PAULA, J.R. ${ }^{1}$ BARA, M.T.F.** \\ ${ }^{1}$ Universidade Federal de Goiás (UFG). Faculdade de Farmácia. Av. Universitária com $1^{a}$ Avenida s/n, Setor \\ Universitário. Caixa Postal 131, CEP: 74605-220. Goiânia-Brasil. *mbara@farmacia.ufg.br, mtbara@gmail.com
}

\begin{abstract}
RESUMO: Trichillia catigua A. Juss. é uma planta medicinal utilizada principalmente como estimulante, possivelmente devido à presença de fenóis. Entre os métodos utilizados para a determinação de polifenóis em matérias-primas vegetais destaca-se a análise por meio de ensaios espectrofotométricos por apresentarem boa sensibilidade, simplicidade, e ter custos mais acessíveis. O presente trabalho teve o objetivo de validar a metodologia analítica para determinação de polifenóis na casca de catuaba em pó. Utilizou-se o método de Hagerman \& Butler para a quantificação dos polifenóis totais encontrando-se 7,96\%. O método validado mostrou-se simples, rápido, seletivo, linear, exato, preciso, e robusto para ser executado na rotina de laboratório de controle de qualidade, constituindo uma possibilidade de análise desta planta medicinal.
\end{abstract}

Palavras-chave: Trichillia catigua, planta medicinal, controle de qualidade, validação.

ABSTRACT: Quantification of total phenols present in the bark of Trichilia catigua A. Juss (Meliaceae). Trichilia catigua A. Juss. is a medicinal plant used especially as a stimulant, possibly due to the presence of phenols. Among the methods used for the determination of polyphenols in raw plant materials is analysis through spectrophotometric assays since they have good sensitivity, are simple and more affordable. This study aimed to validate the analytical methodology for the determination of polyphenols in the bark of powder "catuaba". The method of Hagerman \& Butler was employed in the quantification of total polyphenols, yielding $7.96 \%$. The validated method has proven to be simple, rapid, selective, linear, accurate, precise and robust to be performed in the routine of a laboratory of quality control, constituting a possibility for the analysis of this medicinal plant.

Key words: Trichillia catigua, medicinal plant, quality control, validation

\section{INTRODUÇÃO}

O mercado mundial de fitoterápicos gira em torno de aproximadamente 22 bilhões de dólares, sendo que o mercado europeu e americano alcançou, em 2000, valores de 8,5 e 6,6 bilhões de dólares, respectivamente (Yunes et al., 2001). Desta forma, atraem investimentos no desenvolvimento desses produtos por parte das grandes indústrias farmacêuticas. Segundo Niero (2010), os fitoterápicos representam cerca de 15\% do capital da indústria farmacêutica mundial. No Brasil, esse mercado gira cerca de US\$160 milhões por ano e o fator de atração é o ritmo de crescimento das vendas internas, mais de $15 \%$ anuais, contra $4 \%$ do que evoluem as vendas dos medicamentos sintéticos (Febrafarma, 2009).

Trichillia catigua A. Juss. (Meliaceae), popularmente conhecida como catuaba, é encontrada em florestas semideciduais e em parte da Mata Atlântica. É uma das mais famosas plantas conhecidas como afrodisíacas no Brasil, sendo também utilizada em casos exaustão e como estimulante do sistema nervoso (Albrecht et al., 2007). A ação estimulante se deve provavelmente aos fenóis presentes nas cascas da planta (Beltrame et al., 2006) e da casca de T. catigua detectou-se elevada atividade antioxidante (Brighente et al., 2007).

A T. catigua A. Juss. é uma espécie bioativa muito empregada como matéria-prima industrial e de exportação. Contudo, poucos estudos têm sido publicados sobre a espécie, especialmente quando se tratam de marcadores químicos usados na identificação e nos ensaios de quantificação nas rotinas de controle de qualidade (Lagos, 2006). 
Outro problema de qualidade é relativo à correta identificação de plantas medicinais, podendo-se exemplificar com a espécie Anamopaegma arvense, inscrita na Farmacopéia Brasileira de1998, e que é confundida com a T. catigua, uma vez que ambas são denominadas popularmente como catuaba. A identificação e diferenciação dessas duas espécies é importante, visto que a casca de $T$. catigua é atualmente a mais estudada e possui validação farmacológica (Tobias et al., 2007). Também plantas do gênero Erythroxylum são comumente tratadas como catuaba (Uchino et al., 2004; Beltrame et al., 2006).

Quando se trata de controle de qualidade de plantas medicinais e de fitoterápicos, é de suma importância considerar peculiaridades relativas a fatores genéticos, fisiológicos e ambientais que afetam o desenvolvimento dessas plantas e que influenciam no teor e na sua composição química (Angioni et al., 2003; Masotti et al., 2003; Gobbo Neto \& Lopes, 2007; Botrel et al., 2010; Paula et al., 2011).Vale ressaltar que para determinar o teor dos princípios ativos é necessário desenvolver e validar métodos analiticos que por sua vez darão suporte a atividade farmacológica de uma espécie medicinal (Calixto, 2000).

Embora diversos estudos tenham demonstrado a necessidade de garantir segurança aos produtos de origem vegetal (Brandão et al., 2002; Choi et al., 2002; Bara et al., 2004; Bara et al., 2006), a validação de métodos analíticos para matérias-primas a base de plantas ainda é escassa na literatura (Wang et al., 2003; Ribani et., 2004; Rolim et al., 2005; Lima et al., 2006; Costa et al., 2011; Couto et al., 2011; Sousa et al., 2011; Oliveira et al., 2012). A validação de método analítico tem por objetivo demonstrar que o mesmo é apropriado para a finalidade pretendida, garantindo por meio de estudos experimentais o atendimento aos parâmetros de seletividade, linearidade, intervalo, precisão, limite de detecção, limite de quantificação, exatidão e robustez adequados às análises (Brasil, 2003).

Este trabalho visou aplicar e validar um método analítico para quantificação dos fenóis totais das cascas de $T$. catigua, visto a importância comercial desta planta, seu grande uso em fitoterapia e a necessidade de controlar a sua qualidade.

\section{MATERIAL E MÉTODO}

\section{Amostra de Trichillia catigua}

Foi adquirida uma amostra de $T$. catigua, pó da casca, de fornecedor de matérias-primas idôneo, acompanhada de laudo de identificação da espécie por meio de análises farmacobotânicas e físico-químicas.

Em seguida foi realizada análise cromatográfica por CCD (cromatografia de camada delgada) para investigar a presença de fenóis, marcador da catuaba. Para tanto, foi pesado1g da amostra e colocado sob refluxo em banho-maria $\left(80-90^{\circ} \mathrm{C}\right)$ com $5 \mathrm{~mL}$ de metanol por 10 minutos. Após filtração e concentração do extrato até cerca de $0,5 \mathrm{~mL}$, foram aplicados na cromatoplaca, $20 \mu \mathrm{L}$ da solução extrativa. A fase móvel foi composta por acetato de etila : ácido fórmico : ácido acético glacial : água (100:11:11:26). Após eluição a placa cromatográfica foi observada em luz ultravioleta (UV) a $254 \mathrm{~nm}$ e depois de nebulizada com reagente NP (solução de difenilborato de aminoetanol R a 1\% $\mathrm{p} / \mathrm{v}$ em solução de metanol $\mathrm{R}$ ), observada sob luz UV a $365 \mathrm{~nm}$. Esse ensaio foi adaptado de Wagner \& Bladt (2001).

\section{Análise quantitativa dos fenóis Trichillia catigua}

Foi utilizado o método espectrofotométrico de Hargerman \& Butler para doseamento de fenóis totais, descrito por Waterman \& Mole (1994). Foi pesado $1 \mathrm{~g}$ da amostra, adicionou-se $150 \mathrm{~mL}$ de água destilada e após fervura manteve-se em banho-maria sob refluxo a $80-90^{\circ} \mathrm{C}$ por 30 minutos. O conteúdo foi resfriado e transferido quantitativamente para balão volumétrico de $250 \mathrm{~mL}$. Em seguida o balão foi completado com água destilada e a solução após sedimentação, foi filtrada em papel de filtro. Os primeiros $50 \mathrm{~mL}$ foram desprezados. Essa solução estoque foi empregada na reação cromogênica.

Para o doseamento dos fenóis totais foram adicionados em tubo de ensaio, $2 \mathrm{~mL}$ de solução contendo lauril sulfato de sódio $1 \%$ (LSS), trietanolamina $5 \%$, isopropanol $20 \%, 1 \mathrm{~mL}$ de solução cromogênica de cloreto férrico $\left(\mathrm{FeCl}_{3} 0,162 \%\right)$ e $1 \mathrm{~mL}$ da solução estoque. A mistura foi homogeneizada e após 15 minutos de repouso, foi realizada a leitura da absorbância a 510nm. O branco foi preparado da mesma maneira, substituindo a amostra por água destilada.

Para a construção da curva de calibração foram pesados $25 \mathrm{mg}$ de ácido tânico e transferidos para balão volumétrico de $25 \mathrm{~mL}$, completando-se o volume com água destilada. Alíquotas de 100, 200, 300,400 e $500 \mu \mathrm{L}$ desta solução foram transferidas para tubos de ensaio contendo a solução de LSS/ trietanolamina/ isopropanol e a solução de $\mathrm{FeCl}_{3}$. Em seguida o volume foi completado para $4 \mathrm{~mL}$ com água destilada. Após homogeneização e repouso por 15 minutos, foi realizada a leitura a $510 \mathrm{~nm}$ e obteve a curva. O valor da absorbância foi substituído na variável $y$, da equação $y=a+b x$, obtendo -se a concentração (variável $\mathrm{x}$ ) em mg. $\mathrm{mL}^{-1}$. Com o intuito de determinar a concentração de polifenóis da

Rev. Bras. PI. Med., Campinas, v.15, n.2, p.230-236, 2013. 
catuaba em \%, empregou-se a equação:

$\%$ fenóis $=x\left(\mathrm{mg} \mathrm{mL}^{-1}\right) \cdot 250 \cdot 10^{2} / \mathrm{m}(\mathrm{mg})$

Em que: o número 250 corresponde ao fator de diluição da amostra;

$\mathrm{x}$ : concentração de polifenóis, em mg.mL-1, obtida pela equação da reta

Validação do método analítico empregado

Os ensaios de validação foram baseados na Resolução RE 899/2003 da ANVISA (Agência Nacional de Vigilância Sanitária (Brasil, 2003), que determina o "guia para validação de métodos analíticos e bioanalíticos.

No estudo da validação do método para esta matéria-prima vegetal foram avaliados os parâmetros de seletividade, linearidade, precisão, limite de quantificação, limite de detecção, exatidão e robustez.

\section{a) Precisão}

A amostra foi submetida a 6 determinações a $100 \%$ (contendo a mesma quantidade de amostra preconizada no método), dentro de curto período de tempo e cada doseamento foi realizado em dias diferentes pelo mesmo analista (repetibilidade).

A avaliação da precisão intermediária foi feita através de novas determinações a $100 \%$ por outro analista em 2 dias diferentes.

Conforme a ANVISA a precisão pode ser expressa como desvio padrão relativo (DRP), segundo a fórmula: $\mathrm{DRP}=(\mathrm{DP} / \mathrm{CMD}) \times 100$;

Em que: $\mathrm{DP}=$ desvio padrão

$\mathrm{CMD}$ = concentração média determinada.

\section{b) Exatidão}

Neste trabalho foi verificada a partir de 9 determinações, sendo elas de 3 concentrações diferentes, baixa, média e alta, com 3 réplicas cada.

A exatidão foi calculada conforme equação abaixo:

Exatidão $=$ Concentração obtida $\times 100 /$ Concentração teórica

\section{c) Robustez}

$\mathrm{Na}$ avaliação da robustez variou-se o comprimento de onda para a leitura das absorbâncias, que foram feitas a 508, 510 e $512 \mathrm{~nm}$.

\section{d) Seletividade}

Os espectros de absorção (varredura de comprimentos de onda) da amostra, do branco e do padrão foram realizados na faixa de $200-800 \mathrm{~nm}$.

\section{e) Linearidade}

Foi demonstrada a partir da média de três curvas de calibração. O padrão utilizado foi o ácido tânico, nas concentrações de 0,$1 ; 0,2 ; 0,3 ; 0,4$ e
$0,5 \mathrm{mg} \cdot \mathrm{mL}^{-1}$. $x$ 10/ IC;

f) Limite de Quantificação (LQ)

Foi estabelecido pela equação $L Q=D P a$

Em que: DPa é o desvio padrão do intercepto com o eixo y de 3 curvas de calibração construídas contendo concentrações do fármaco próximas ao suposto limite de quantificação; e IC é a inclinação da curva de calibração.

\section{g) Limite de Detecção (LD)}

Foi determinado pela equação $L D=D p a x$ 3/ IC; Em que: DPa é o desvio padrão do intercepto com o eixo y de 3 curvas de calibração construídas contendo concentrações do fármaco próximas ao suposto limite de quantificação.

\section{RESULTADO E DISCUSSÃO}

Atualmente, as plantas representam alternativa entre as diversas fontes de insumos necessários à existência do homem, tendo como principal vantagem o fato de ser fonte renovável e, em grande parte, controlada pelo ser humano, além de ter custo mais acessível à população e aos serviços públicos de saúde se comparado aos medicamentos obtidos por síntese química (Farias, 2004).

\section{Caracterização da amostra estudada}

A cromatografia de camada delgada (CCD) permitiu a visualização de quatro bandas, sendo uma banda azulada fluorescente com $\mathrm{Rf}=0,21$ - indicativa de fenóis e três bandas amareladas fluorescentes com valores de $\operatorname{Rf} 0,44 ; 0,55$ e 0,73 , indicativas de flavonóides da catuaba, após revelação com NP.

Não há na literatura especificação do perfil cromatográfico da espécie Trichilia catigua, por isso utilizou-se como método a referência geral para fenóis totais (Wagner \& Bladt, 2001). A presença de fenóis nesta espécie foi descrita por Beltrame et al. (2006) e de flavonóides por Rolim et al. (2005).

\section{catigua \\ Análise quantitativa dos fenóis da Trichilia}

O método espectrofotométrico de Hargerman \& Butler para doseamento de fenóis totais, descrito por Waterman \& Mole (1994) foi selecionado pela simplicidade, rapidez e custos baixos. Esse ensaio colorimétrico fundamenta-se na reação de compostos fenólicos com $\mathrm{FeCl}_{3}$, resultando na formação de complexos que absorvem a $510 \mathrm{~nm}$.

Os resultados demonstraram valor médio de $7,96 \%$ de fenóis totais na casca da catuaba (Tabela 1). 
TABELA 1. Teor de fenóis totais em Trichillia catigua A. Juss., pelo método espectrofotométrico.

\begin{tabular}{ccc}
\hline Amostras & Absorbância & Concentração (\%) \\
\hline 1 & 0,721 & 8,39 \\
2 & 0,711 & 8,26 \\
3 & 0,676 & 7,89 \\
4 & 0,679 & 7,92 \\
5 & 0,676 & 7,89 \\
6 & 0,676 & 7,90 \\
7 & 0,694 & 8,10 \\
8 & 0,690 & 8,06 \\
9 & 0,657 & 7,66 \\
10 & 0,647 & 7,54 \\
\hline Média & 0,6827 & 7,961 \\
\hline DP & 0,02244 & 0,255145 \\
\hline DRP & 3,286 & 3,20 \\
\hline
\end{tabular}

É importante ressaltar que para um fitoterápico ser considerado seguro e eficaz, ele deve ser avaliado quanto às qualidades físico-químicas e microbiológicas, como etapa preliminar para alcançar um padrão adequado (Bara et al., 2006). Além disso, por meio do estabelecimento de parâmetros de qualidade para a matéria-prima, a qualidade do produto final (medicamento) estará, em grande parte, assegurada. Portanto, a qualidade da matériaprima vegetal é a determinante inicial da qualidade do fitoterápico, mas não garante por si só a qualidade final do produto (Fischer et al., 2010).

Validação do método analítico empregado Por não terem sido encontradas monografias da Trichilia catigua nas farmacopéias pesquisadas (Farmacopéia Brasileira, IV edição, 1988-2006; United States Pharmacopoea 24-28, 2000-2005; European Pharmacopoea 1997, 2001; British Pharmacopoea, 2000, 2004), nesse estudo o método geral descrito para análise de fenóis (Waterman \& Mole, 1994) foi aplicado e validado.

\section{a) Precisão}

A precisão é a avaliação da proximidade dos resultados obtidos em uma série de medidas de amostragem múltipla de mesma amostra. É considerada em três níveis, a repetibilidade, precisão intermediária e reprodutibilidade, essa última realizada em laboratórios diferentes (Brasil, 2003).

Neste trabalho avaliou-se primeiramente, a repetibilidade (Tabela 2), considerada o parâmetro mais importante pela USP (United States
TABELA 2. Precisão por repetibilidade do método para doseamento dos fenóis totais de Trichillia catigua A. Juss.

\begin{tabular}{ccc}
\hline Amostras & Absorbância & Concentração (\%) \\
\hline 1 & 0,759 & 8,89 \\
2 & 0,740 & 8,66 \\
3 & 0,702 & 8,19 \\
4 & 0,769 & 9,01 \\
5 & 0,735 & 8,59 \\
6 & 0,699 & 8,16 \\
\hline Média & 0,6827 & 8,58 \\
\hline DP & 0,02244 & 0,350 \\
\hline DRP & 3,286 & 4,089 \\
\hline
\end{tabular}

Pharmacopoeae) 24, 2000.

Pode-se observar que o método tem boa repetibilidade, visto que o desvio padrão relativo (DRP) foi inferior ao especificado pela ANVISA, que é de $5 \%$. Deve-se ressaltar que a validação de método analítico para fitoterápicos difere dos fármacos sintéticos devido à complexidade da matéria-prima vegetal. Sendo assim, a validação de metodologia analítica para fitoterápicos deve ser feita de acordo com o disposto na RE 899/03, porém, os resultados podem seguir os níveis de

TABELA 3. Precisão Intermediária do método para doseamento dos fenóis totais de Trichillia catigua A. Juss.

\begin{tabular}{ccc}
\hline Analistas & Absorbância & Concentração (\%) \\
\hline 1 & 0,697 & 7,52 \\
1 & 0,685 & 7,43 \\
Média & 0,691 & 7,48 \\
\hline 2 & 0,680 & 7,37 \\
2 & 0,650 & 7,02 \\
Média & 0,665 & 7,2 \\
\hline Média & 0,678 & 7,34 \\
\hline DP & 0,01838 & 0,19799 \\
\hline DRP & 2,71 & 2,69 \\
\hline
\end{tabular}

aceitação estipulados para métodos bioanalíticos, considerando-se tal complexidade pode-se aceitar uma dispersão de $15 \%$ em todos os pontos (BRASIL, 2010).

A precisão intermediária foi avaliada a partir de determinações feitas por analistas diferentes e os resultados encontram-se na Tabela 3. Pode-se verificar que não houve diferença superior a $5 \%$, entre os ensaios realizados em dias diferentes e entre analistas. 
TABELA 4. Exatidão do método para doseamento dos fenóis totais de Trichillia catigua A. Juss.

\begin{tabular}{lccc}
\hline \multicolumn{3}{l}{ AmostrasAbsorbâncias Concentração } & $\begin{array}{c}\text { Exatidão } \\
(\%)\end{array}$ \\
\hline $1(50 \%)$ & 0,381 & 7,79 & \\
$2(50 \%)$ & 0,351 & 7,14 & 95,1 \\
$3(50 \%)$ & 0,376 & 7,69 & \\
Média & 0,369 & 7,57 & \\
\hline $4(100 \%)$ & 0,685 & 7,33 & 92,6 \\
$5(100 \%)$ & 0,689 & 7,36 & \\
$6(100 \%)$ & 0,690 & 7,4 & \\
Média & 0,688 & 7,37 & 95,3 \\
\hline $7(150 \%)$ & 1,046 & 7,6 & \\
$8(150 \%)$ & 1,043 & 7,56 & \\
$9(150 \%)$ & 1,046 & 7,60 & \\
\hline Média & 1,045 & 7,59 & \\
\hline Média & 7,51 & & \\
\hline
\end{tabular}

\section{b) Exatidão}

A exatidão é a proximidade dos resultados obtidos pelo método em estudo em relação ao valor verdadeiro e pode ser determinada através de várias metodologias.

A exatidão do método foi comprovada pelo estudo de três concentrações: baixa, média e alta (50, 100 e $150 \%)$ e o percentual de recuperação para as três concentrações de foi de 95,1, 92,6 e $95,3 \%$, respectivamente (Tabela 4 ).

TABELA 5. Robustez do método para doseamento dos fenóis totais Trichillia catigua A. Juss.

\begin{tabular}{lccc}
\hline $\begin{array}{l}\text { Comprimento } \\
\text { de onda }\end{array}$ & $\begin{array}{c}\text { Média das } \\
\text { Absorbâncias }\end{array}$ & $\begin{array}{c}\text { Concentração } \\
\%\end{array}$ & Teor \\
\hline $508 \mathrm{~nm}$ & 0,678 & 7,32 & 92 \\
$510 \mathrm{~nm}$ & 0,682 & 7,32 & 92 \\
$512 \mathrm{~nm}$ & 0,689 & 7,40 & 93 \\
\hline
\end{tabular}

A
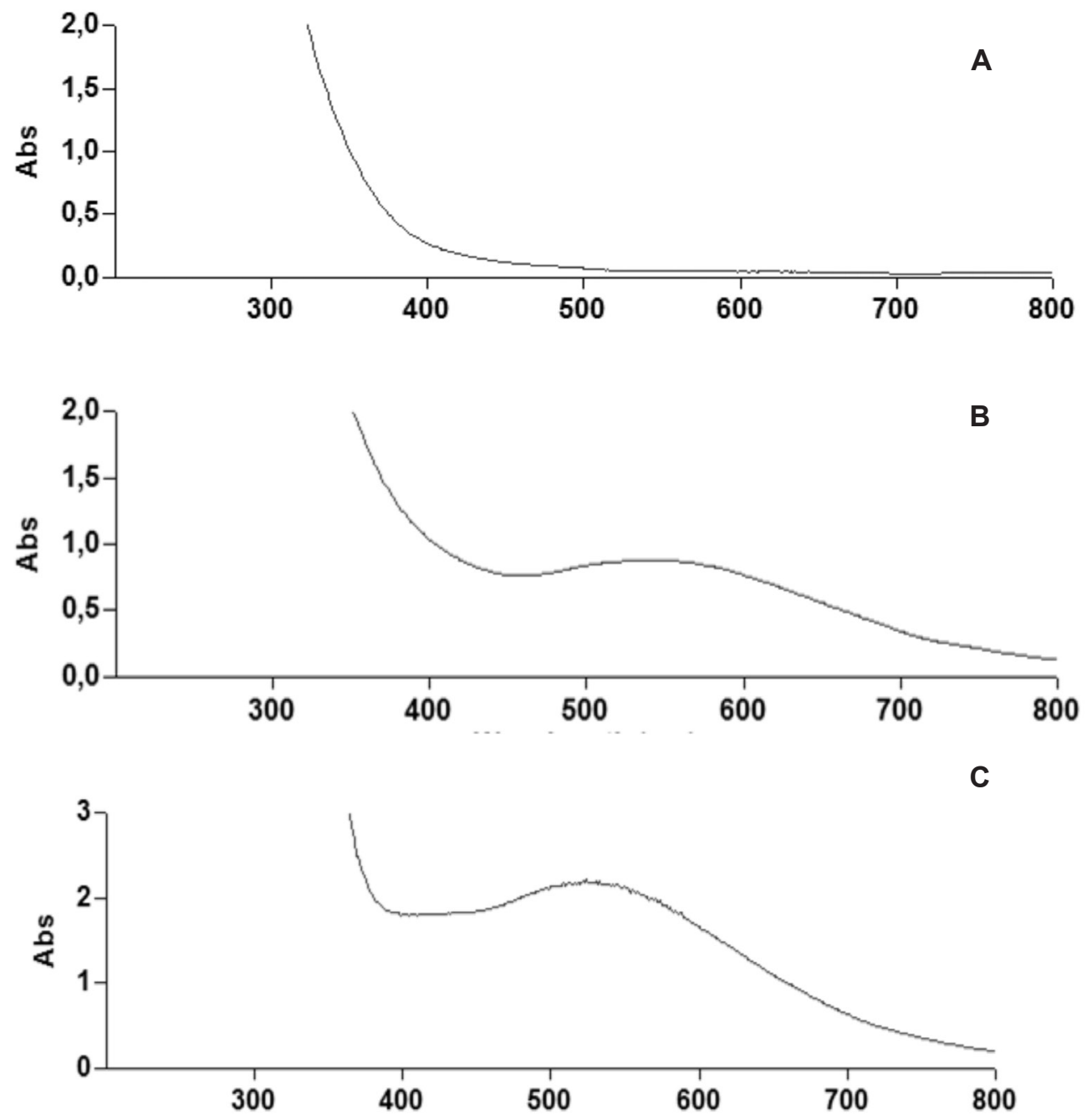

FIGURA 1. Espectro de absorção (varredura na faixa de 200 - $800 \mathrm{~nm}$ ) para avaliação do parâmetro seletividade: A) branco B) amostra (Trichillia catigua A. Juss.), C) padrão (ácido tânico).

Rev. Bras. PI. Med., Campinas, v.15, n.2, p.230-236, 2013. 


\section{c) Robustez}

A robustez é o parâmetro que verifica a capacidade que o método analítico apresenta em fornecer resultados inalterados quando sujeito a pequenas mudanças.

A avaliação da robustez foi realizada através da variação do comprimento de onda (Tabela 5). Podese verificar que a variação do comprimento de onda não interferiu nos resultados.

\section{d) Seletividade}

É a capacidade que o método possui de medir exatamente um composto em presença de outros componentes tais como impurezas, produtos de degradação e outras substâncias.

Os resultados da varredura do branco, da amostra e do padrão estão demonstrados nas Figuras $1 \mathrm{~A}-\mathrm{C}$, respectivamente.

Pode-se perceber que o branco não interfere na leitura da amostra e essa, por sua vez, produziu um pico de absorção na faixa de 510 a $546 \mathrm{~nm}$, assim como o padrão de ácido tânico.

\section{e) Linearidade}

É a capacidade de uma metodologia analítica de demonstrar que os resultados obtidos são diretamente proporcionais à concentração do analito na amostra, dentro de intervalo especificado. Para esse método, trabalhamos com concentrações de catuaba de 0,1 a $0,5 \mathrm{mg} / \mathrm{mL}$ e obtivemos as respectivas absortividades: 0,264-0,486- 0,717-0,938- 1,165. Assim, na análise de regressão linear encontramos a equação $y=2,254 x+0,037$ e um coeficiente de correlação $r=1,000$, valor que comprova a linearidade do método, nas condições empregadas.

\section{f) Limite de Quantificação e Detecção}

Limite de Quantificação é a menor quantidade do analito em amostra que pode ser determinada com precisão e exatidão aceitáveis. Não é um parâmetro exigido para validação de produtos da categoria I, mas foi analisado neste trabalho.

Limite de Detecção é a menor quantidade do analito presente em uma amostra que pode ser determinado, porém não necessariamente quantificado.

Os limites de detecção $\left(0,0006 \mathrm{mg} \mathrm{mL}^{-1}\right) \mathrm{e}$ de quantificação $\left(0,003 \mathrm{mg} \mathrm{mL}^{-1}\right)$ obtidos demonstram que o método é sensível.

$\mathrm{Na}$ literatura científica há descrição desse mesmo método espectrofotométrico para quantificar polifenóis em plantas medicinais (Sampaio et al., 2011; Borges et al., 2012; Alcântara et al., 2012) e outro estudo descreveu método semelhante (Brighenti et al., 2007), entretanto a validação analítica não foi demonstrada. Além disso, encontrou-se um trabalho que emprega a cromatografia líquida de alta eficiência para a análise da catuaba (Beltrame et al., 2006), porém, embora seja esse um método analítico mais sensível e específico, é de custo mais elevado.

Por fim, destaca-se a grande importância da validação de métodos analíticos aplicados às plantas medicinais e fitoterápicos visando contribuir para a homogeneizar os teores de princípios ativos presentes numa determinada espécie, o que por sua vez influenciará na atividade farmacológica dessa planta medicinal, no sentido de permitir utilizar as dosagens terapêuticas padronizadas.

\section{CONCLUSÃO}

Os resultados da validação do método para determinação do teor de fenóis totais da T. catigua comprovaram que este é seletivo, linear, preciso, exato e robusto. Portanto, representa uma possibilidade para a quantificação desta classe química, uma vez que apresenta a confiabilidade requerida para um método analítico, além de ser barato, simples e rápido.

\section{AGRADECIMENTO}

Os autores agradecem ao Conselho Nacional de Desenvolvimento Científico e Tecnológico (CNPq) pelo apoio financeiro.

\section{REFERÊNCIA}

ALBRECHT, I.; OLIVEIRA, F.R.; MELLO, J.P.C. Atividade antioxidante de extratos e substâncias isoladas de cascas de Trichilia catigua A. Juss. In: CONGRESSO INTERNACIONAL DE SAÚDE, 2, 2007, Maringá. Anais... Maringá: UEM, 2007. p. 21.

ALCÂNTARA, G.A.; BORGES, L.L.; PAULA, J.R. Seasonal variation in the content of phenolic compounds in barks of Myrcia rostrata DC. by influence of environmental factors. Journal of Pharmacy Research, v.5, n.3, p.1306-9, 2012.

ANGIONI, A. et al. Chemical composition, seasonal variability, and antifungal activity of Lavandula stoechas L. ssp. stoechas essential oils from stem/leaves and flowers. Journal of Agricultural and Food Chemistry, v.54, n.12, p.4364-70, 2003.

BARA, M.T.F. et al. Determinação de ginkgoflavonóides por cromatografia líquida de alta eficiência em matériasprimas e produtos acabados. Revista Eletrônica de Farmácia, v.1, n.1, p.1-4, 2004.

BARA, M.T.F. et al. Determinação do teor de princípios ativos em matérias-primas vegetais. Revista Brasileira de Farmacognosia, v.16, n.2, p. 211-5, 2006.

BELTRAME, F.L. et al. A validated higher-performance liquid chromatography method for quantification of cinchonain Ib in bark and phytopharmaceuticals of Trichilia catigua used as Catuaba. Journal of Chromatography A, v.1119, p.257-63, 2006.

BORGES, L.L. et al. Influence of environmental factors on the concentration of phenolic compounds in barks of Myrcia tomentosa (Aubl.) DC. Journal of Pharmacy Research, v.5, n.3, p.1323-27, 2012. 
BOTREL, P.P. et al. Variações no teor e na composição de Hyptis marrubioides EPL. Cultivado no campo e em casa de vegetação. Química Nova, v.33, n.1, p.33-7, 2010.

BRANDÃO, M.G.L. et al. Qualidade de amostras comerciais de chás de plantas medicinais. Revista Brasileira de Plantas Medicinais, v.5, n.1, p.56-9, 2002.

BRASIL. Ministério da Saúde. Agência Nacional de Vigilância Sanitária. Resolução - RE n. 899. Guia para Validação de Metodologias Analíticas, 2003.

BRASIL, Orientações sobre "Controle de qualidade de extratos vegetais e fitoterápicos, 2010. Disponível em: <http://www.anvisa.gov.br/medicamentos/fitoterapicos/ Controle_qualidade_extratos.pdf>.

Acesso em: 8 set. 2010.

BRIGHENTE, I.M.C. et al. Antioxidant Activity and Total Phenolic Content of Some Brazilian Species. Pharmaceutical Biology, v. 45, n. 2, p. 156-61, 2007.

BRITISH PHARMACOPOEIA, London: British Pharmacopoeia Commission, Her Majesty's Stationery Office. 2000.

BRITISH PHARMACOPOEA, 2004. London: Stationery Office Inc. (cd rom).

CALIXTO, J.B. Efficacy, safety, quality control, marketing and regulatory guidelines for herbal medicines (phytotherapeutic agent). Brazilian Journal of Medical and Biological Research, v. 33, n.2, p. 179-89, 2000.

$\mathrm{CHOI}, \mathrm{D} . W$. et al. Regulation and quality control of herbal drugs in korea. Toxicology, v.181-182, p.581-6, 2002.

COSTA, A.R.T. et al. Dissolution test of herbal medicines containing Passiflora sp. Brazilian Journal of Pharmacognosy, v.21, n.3, p.525-31, 2011.

COUTO, R.O. et al. Validated HPLC-PDA method for rosmarinic acid quantification in Rosemary. Latin American Journal of Pharmacy, v. 30, n.10, p.1951-6, 2011.

EUROPEAN PHARMACOPOEA, Strasbourg: Council of Europe. 1997.

EUROPEAN PHARMACOPOEA, Strasbourg: Council of Europe. 2001.

FARIAS, M.R. Avaliação da qualidade de matérias-primas vegetais. In: SIMÕES, C.M.O. et al. Farmacognosia da planta ao medicamento. $5^{\mathrm{a}}$ ed. Porto Alegre: Editora UFRGS, 2004. p.263-88.

FARMACOPÉIA BRASILEIRA: $4^{\mathrm{a}}$ ed. São Paulo: Editora Atheneu. 1988 - 2005.

FEBRAFARMA. Disponível em:<http// www.febrafarma.org. br>. Acesso em: 05 nov. 2009.

FISCHER, D.C.H.; BARA, M.T.F.; SILVEIRA, D. Controle de qualidade de fitoterápicos. In: GIL, E.S. Controle físicoquímico de qualidade de medicamentos. 3.ed. São Paulo: Editora Pharmabooks, 2010, p.297-348.

GOBBO NETO, L., LOPES, N.P. Plantas Medicinais: fatores de influência no conteúdo de metabólitos secundários. Quimica Nova, v.30, n.2, p.374-81, 2007.

LAGOS, J.B. Estudo Comparativo da composição química das folhas e cascas da Trichilia catigua A. Juss., Meliaceae. 2006. 116 p. Dissertação (Mestrado em Ciências Farmacêuticas). Universidade Federal do Paraná, Curitiba.

LIMA, L.R. et al. Desenvolvimento e validação da metodologia de quantificação gravimétrica de resina glicosídica em fitoterápicos contendo Operculina macrocarpa (L.) Urban. Revista Brasileira de Farmacognosia, v.16, n.4, p. 562-7, 2006.

MASOTTI, V. et al. Seasonal and phenological variations of the essential oil from the narrow endemic species Artemisia molinieri and its biological activities. Journal of Agricultural and Food Chemistry, v. 51, n.24, p. 7115-21, 2003.

NIERO, R. Fármacos, fitofármacos e fitoterápicos: abordagem econômica e de mercado. In: BRESOLIN, T. M. B.; CECHINEL FILHO, V. Fármacos e medicamentos: uma abordagem multidisciplinar. 1.ed. São Paulo: Santos, 2010, p.1-15.

OLIVEIRA, P.C. et al. Obtaining a dry extract of Pterodon emarginatus (Fabaceae) fruits by spray-drying. Journal of Pharmacy Research, v. 5, n.1, p.641-5, 2012.

PAULA, J.A.M. et al. Infraspecific chemical variability in the essential oils of Pimenta pseudocaryophyllus (Gomes) L.R. Landrum (Myrtaceae). Biochemical Systematics and Ecology, v. 39, n.4-6, p.643-50, 2011.

ROLIM, A. et al. Validation assay for total flavonoids, as rutin equivalents, from Trichilia catigua A. Juss (Meliaceae) and Ptychopetalum olacoides Bentham (Olacaceae) commercial extract. Journal of AOAC International, v.88, n.4, p.1015-9, 2005.

RIBANI, M. et al. Validação em métodos cromatográficos e eletroforéticos. Química Nova, v.27, n.5, p.771-80, 2004.

SAMPAIO, B.L. et al. Influence of environmental factors on the concentration of phenolic compounds in leaves of Lafoensia pacari. Revista Brasileira de Farmacognosia, v. 21, n 6, p. 1127-37, 2011.

SOUSA, S.A. et al. Dissolution test of herbal medicines containing Paullinia cupana: validation of methods for quantification and assessment of dissolution. Brazilian Journal of Pharmaceutical Sciences, v.47, n. 2, p.26977, 2011.

TOBIAS, M.L. et al. Controle de qualidade de drogas vegetais de farmácias de manipulação de Maringá. Revista Eletrônica de Farmácia, v.4, n.1, p.95-103, 2007.

UCHINO, T. et al. Potent protecting effects of catuaba (Anemopaegma mirandum) extracts against hydroperoxideinduced cytotoxicity. Toxicology in vitro, v.18, n.3, p.25563, 2004.

UNITED STATES PHARMACOPOEA - The National Formulary - NF 24, Rockville, 2000.

UNITED STATES PHARMACOPOEA - The National Formulary - NF 25, Rockville, 2001.

UNITED STATES PHARMACOPOEA - The National Formulary - NF 26, Rockville, 2002.

UNITED STATES PHARMACOPOEA - The National Formulary - NF 27, Rockville, 2003.

UNITED STATES PHARMACOPOEA - The National Formulary - NF 28, Rockville, 2004.

UNITED STATES PHARMACOPOEA - The National Formulary - NF 29, Rockville, 2005.

YUNES, R.A.; PEDROSA, R.C.; FILHO, V.C. Fármacos e fitoterápicos: a necessidade do desenvolvimento da indústria de fitoterápicos e fitofármacos no Brasil. Química Nova, v.24, n.1, p.147-52, 2001.

WAGNER, H.; BLADT, S. Plant drug analysis. $2^{a}$ ed. New York: Springer, 2001. 384p.

WANG, F.M.; YAO, T.W.; ZENG, S. Disposition of quercetin and kaempferol in human following an oral administration of Ginkgo biloba extract tablets. European Journal of Drug Metabolism and Pharmacokinetics, v.28, n.3, p.173-7, 2003.

WATERMAN, P.G.; MOLE, S. Analysis of phenolic plant metabolites. Oxford: Blackwell. Scientific Publication, 1994. 238p. 